\title{
Uterus like ovarian mass in a young female clinically mimicking Carcinoma ovary
}

\author{
Jaydeep Pol ${ }^{1}$, Yash Kale ${ }^{2, *}$, Madhura Phadake ${ }^{3}$, Girish Kadkol ${ }^{4}$ \\ Consultant Pathologist, The Oncopathology Center, Mahatma Gandhi Cancer Hospital, Miraj, Maharashtra, India
}

*Corresponding Author:

Email: yashkale33@gmail.com

\begin{abstract}
Uterus - like ovarian mass is a rare entity of uncertain histogenesis. A 30 year old female had a Left ovarian mass. Her preoperative serum CA 125 was 700U/ml. So a clinical diagnosis of Carcinoma ovary was made. We received left salpingoopherectomy specimen for intraoperative frozen consultation. On gross examination, left ovarian mass measured $10 x 8 x 6.5 \mathrm{~cm}$. External surface was smooth and showed congested blood vessels. On cutting, mass was partly solid and partly cystic showing small cystic cavities filled with blood mixed fluid. The cyst wall surrounding the cavities was remarkably thickened. Microscopic examination of frozen section revealed a lesion predominantly composed of benign spindle cells. So we gave a diagnosis of benign cystic non neoplastic lesion on frozen section. Formalin fixed paraffin embedded sections followed. Microscopic examination of formalin fixed paraffin embedded sections revealed a cystic mass lined by endometrial type of lining and stroma surrounded by remarkable smooth muscle hyperplasia. In areas, haemorrhage, infiltration by hemosiderophages and hemorrhagic corpus luteum were also seen. So we gave a final diagnosis of uterus like ovarian mass.

To the best of our knowledge, only 15 cases of extrauterine uterus like masses have been reported in the literature. Of these, 5 cases were of ovarian origin. This report documents a sixth case of uterus-like ovarian mass.
\end{abstract}

Keywords: Uterus like ovarian mass in a young female clinically mimicking Carcinoma ovary.

\section{Introduction}

Uterus - like ovarian mass is a rare entity of uncertain histogenesis. Uterus like extra uterine-masses within the pelvis may arise in the broad ligament, fallopian tube, or ovary ${ }^{1}$. It represents a unique arrangement of a cavity lined by endometrial mucosa surrounded by bundles of smooth muscle fibres, replacing the normal structure of ovary. To the best of our knowledge, only 15 cases of extrauterine uterus like masses have been reported in the literature ${ }^{2}$. Of these, 5 cases were of ovarian origin..$^{3-7}$ This report documents a sixth case of uterus-like ovarian mass.

\section{Case Report}

A 30 year old female had a Left ovarian mass. Her preoperative serum CA 125 was $700 \mathrm{U} / \mathrm{ml}$. So a clinical diagnosis of Carcinoma ovary was made. We received left salpingo-opherectomy specimen for intraoperative frozen consultation. On gross examination, left ovarian mass measured $10 \times 8 \times 6.5 \mathrm{~cm}$. External surface was smooth and showed congested blood vessels. On cutting, mass was partly solid and partly cystic showing small cystic cavities filled with blood mixed fluid. The cyst wall surrounding the cavities was remarkably thickened (Fig. 1). Microscopic examination of frozen section revealed a lesion predominantly composed of benign spindle cells. So we gave a diagnosis of benign cystic non neoplastic lesion on frozen section.

Formalin fixed paraffin embedded sections followed. Microscopic examination of formalin fixed paraffin embedded sections revealed a cystic mass lined by endometrial type of lining and stroma surrounded by remarkable smooth muscle hyperplasia (Fig. 2). In areas, haemorrhage, infiltration by hemosiderophages and hemorrhagic corpus luteum were also seen. So we gave a final diagnosis of uterus like ovarian mass.

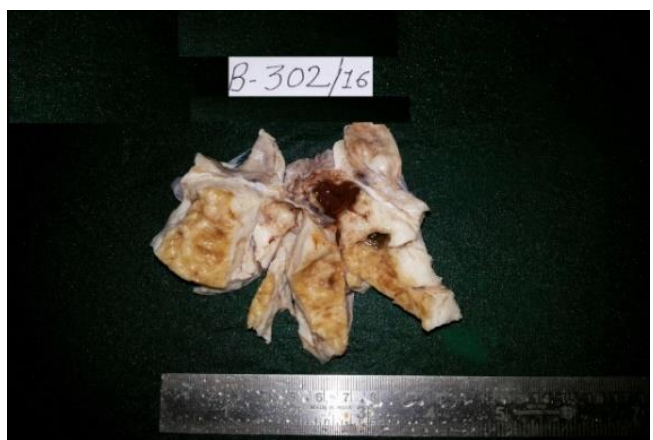

Fig. 1: Gross specimen of ovarian mass showing small cystic cavities containing hemorrhagic fluid. Cyst wall surrounding the cavities is remarkably thickened

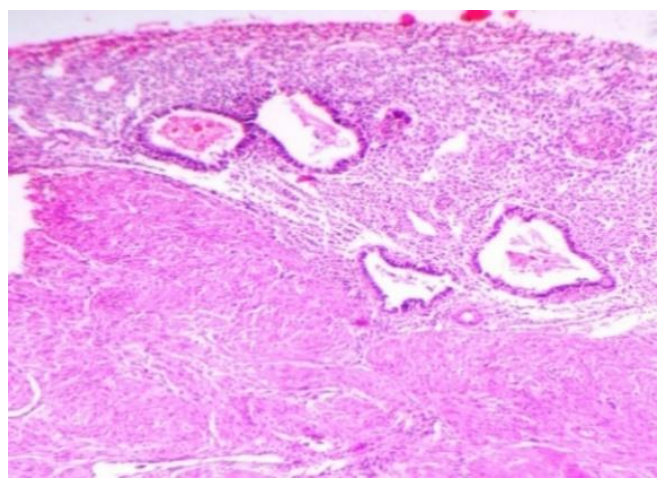

Fig. 2: Cyst wall lined by endometrial glands and stroma. Wall shows marked smooth muscle proliferation 


\section{Discussion}

The Uterus like ovarian masses are extremely rare. The first uterus - like mass was described by Cozzzutto $^{3}$ in 1981 . Since then only 15 cases have been published in the literature. ${ }^{2}$ Of these 12 cases were located within pelvis and 5 cases arising in the ovaries. ${ }^{2-7}$ Rare cases originating from broad ligament, liver and mesentry have been reported., ${ }^{2,-10}$. Two extraovarian cases were reported at pelvic location after hysterectomy. ${ }^{1,11}$

Sizes ranged from 6 to $16 \mathrm{~cm}$. The gross appearance, including cystic and solid areas, varied between cases. ${ }^{6,7}$ In our case, size was $10 \times 8 \times 6.5 \mathrm{~cm}$ and on gross examination of the mass was solid and cystic in appearance.

The microscopic descriptions of each mass included functional endometrial glands and stroma within a muscular walled structure resembling a uterus. In our case microscopic appearance also revealed same thing.

Ovarian endometriosis is associated with elevated serum CA 125 levels. In our case raised CA 125 levels were probably due to ovarian endometriosis. ${ }^{12}$

The pathogenesis of uterus-like masses remains uncertain. When we reviewed literature, we found out four theories to explain this occurance. The first theory is mullerian duct fusion defect theory is based on a developmental abnormality occurring during the formation of the female genital tract. ${ }^{13-15}$ The second theory is sub-coelomic mesenchyme transformation. Third is mullerianosis theory defined by Batt as a heterotopic organoid structure composed of Müllerian rests that were incorporated within other normal organs during organogenesis. ${ }^{18,19}$

Endometriosis with prominent smooth muscle hyperplasia or metaplasia, which has also been called endomyometriosis, is the fourth theory to explain pathogenesis. Pathogenesis in our case is best explained by the fourth theory. ${ }^{20,21}$

\section{Conclusion}

In conclusion, these rare lesions present with ovarian mass having raised CA 125 levels. Hence, they are clinically mistaken for $\mathrm{Ca}$ ovary. Therefore, one must diagnose such lesions precisely, as they are benign and just require surgical excision. In the coming years, we are likely to encounter such lesions during intraoperative frozen sections as in our case.

\section{References}

1. Redman R, Wilkinson EJ, Massoll NA: Uterine-like mass with features of an extrauterineadenomyoma presenting 22 years after total abdominal hysterectomy-bilateral salpingo-oophorectomy: a case report and review of the literature. Arch Pathol Lab Med 2005,129:1041-3.

2. Huanwen et al.: Extrauterineadenomyoma of the liver with a focally cellular smooth muscle component occurring in a patient with a history of myomectomy: case report and review of the literature. Diagnostic Pathology 2013;8:131.

3. Cozzutto C. Uterus like mass replacing ovary. Arch Pathol Lab Med. 1981;105:508-11.

4. Pueblitz-Peredo S, Luevano-Flores E, Rincon-Taracena R, Ochoa-Carrillo FJ. Uterus-like mass of the ovary: endometriosis or congenital malformation. Arch Pathol Lab Med. 1985;109:361-4.

5. Rahilly MA, Al-Nafussi A. Uterus-like mass of the ovary associated with endometrial carcinoma. Histopathology. 1991;18:549-51.

6. Pai SA, Desai SB, Borges AM. Uterus like masses of ovary associated with breast cancer and raised serum CA 125. AM J Surg Pathol 1998;22:333-7

7. Shutter J . Uterus like mass presenting near menarche. Int J of GynecolPathol 2005;24:382-4.

8. Ahmed AA, Swan RW, Owen A, Kraus FT, Patrick F. Uterus-like mass arising in the broad ligament: a metaplasia or Mullerian duct anomaly? Int J Gynecol Pathol. 1997;16:279-81.

9. Liang YJ, Hao Q, Wu YZ, Wu B: Uterus-like mass in the left broad ligament misdiagnosed as a malformation of the uterus: a case report of a rarecondition and review of the literature. FertilSteril 2010,93(1347)

10. Horie Y, Kato M. Uterus-like mass of the small bowel mesentery. PatholInt 2000;50:76-80.

11. Khurana A, Mehta A, Sardana M.

Extrauterineadenomyoma with uterus like features: A rare entity presenting 17 years post hysterectomy. Indian J PatholMicrobiol 2011;54:572-3.

12. Jo Kitawaki1, Hiroaki Ishihara, HisatoKoshiba, Miyo Kiyomizu, Mariko Teramoto, YuiKitaoka and Hideo Honjo Usefulness and limits of CA-125 in diagnosis of endometriosis without associated ovarian endometriomas Human Reproduction Vol.20, No.7 pp. 1999-2003, 2005.

13. SadlerTW. Langman's Medical Embryology. 6th ed. Baltimore, Md:Williams and Wilkins; 1990:270-283.

14. Lauchlan SC. The secondary Mulleriansystem.ObstetGynecolSurv. 1972;27:133-46.

15. Rosai J. Uterus-like mass replacing ovary [letter]. Arch Pathol Lab Med. 1982;106:364.

16. Ober WB, Black MB. Neoplasms of the subcoelomicmesenchyme.ArchPathol. 1955;59:698-705.

17. Kurman RJ. Blaunstein's Pathology of the Female Genital Tract. 5th ed. New York, NY: springer-Verlag; 2002:745-78.

18. Batt RE, Smith RA, Buck Louis GM, Martin DC, Chapron C, Koninckx PR, Yeh J: Mullerianosis. HistolHistopathol 2007, 22:1161-6.

19. Batt RE: Pathogenesis of a parauterine uterus-like mass: developmentally misplaced mullerian tissuemullerianosis. FertilSteril 2010,94:e45.

20. Clement PB: The pathology of endometriosis: a survey of the many faces of a common disease emphasizing diagnostic pitfalls and unusual and newly appreciated aspects. Adv Anat Pathol 2007,14:241-60.

21. Matsuzaki S, Murakami T, Sato S, Moriya T, Sasano H, Yajima A: Endomyometriosis arising in the uterosacral ligament: a case report including a literature review and immune histochemical analysis. PatholInt 2000, 50:4936. 